\title{
Metformin use in patients with type 2 diabetes mellitus is associated with reduced risk of deep vein thrombosis: a non-randomized, pair-matched cohort study
}

\author{
Dai-Yin Lu', Chin-Chou Huang 1,2,3,4 , Po-Hsun Huang ${ }^{1,3,5}$, Chia-Min Chung ${ }^{6}$, Shing-Jong Lin 1,2,3,5, \\ Jaw-Wen Chen 1,2,3,5, Wan-Leong Chan ${ }^{1,7}$ and Hsin-Bang Leu ${ }^{1,2,3,5,7^{*}}$
}

\begin{abstract}
Background: Metformin, an insulin-sensitizer, may correct several physiologic abnormalities owing to insulin resistance in patients with type 2 diabetes mellitus (DM). The effects of metformin on venous thrombosis in patient with type 2 DM have not been reported. Our study strived to explore the relationship of metformin therapy and the subsequent development of deep vein thrombosis (DVT) using a nationwide, population-based database.

Methods: From 1997 to 2003, we identified a study cohort consisting of patients with type 2 DM using metformin 7154 cases in the National Health Insurance Research Database. A control cohort without metformin, matched for age, sex, comorbidities, and medications was selected for comparison.

Results: Of the 14945 patients (7167 patients with metformin vs. 7778 control), 60 (0.40\%) patients developed DVT during a mean follow-up period of 3.74 years, including $16(0.21 \%)$ from the cohort with metformin and $44(0.56 \%)$ from the control group. Subjects with metformin experienced a 0.427 fold (95\% confidence interval 0.240-0.758; $P=0.004)$ changes of risk reduction in development of DVT, which was independent of age, sex and co-morbidities. Kaplan-Meier analysis also revealed metformin therapy is associated with lower occurrence of DVT (log-rank test, $P=0.001$ ).
\end{abstract}

Conclusions: Metformin may have protective effect in patients with type 2 DM for DVT.

Keywords: Deep vein thrombosis, Metformin, Type 2 diabetes mellitus

\section{Background}

Vascular disease is a major cause of morbidity and mortality among patients with diabetes, and these patients account for a significant proportion of all patients with cardiovascular disease, including coronary artery disease, acute myocardial infarction, and cerebral infarction [1]. Insulin resistance contributes greatly to development of cardiovascular disease in patient with metabolic syndrome and type 2 diabetes mellitus (DM). Therefore, treatment with an insulin-sensitizing agent, such as

\footnotetext{
* Correspondence: hbleu@vghtpe.gov.tw

'Division of Cardiology, Department of Medicine, Taipei Veterans General Hospital, Taipei, Taiwan

2Department of Medical Research and Education, Taipei Veterans General Hospital, Taipei, Taiwan

Full list of author information is available at the end of the article
}

metformin may correct several of the primary pathophysiologic abnormalities, including lipid metabolism, endothelial function, and platelet hyperactivity in patients with diabetes mellitus [2]. The United Kingdom Prospective Diabetes Study Group (UKPDS) has shown that patients with type $2 \mathrm{DM}$ treated with metformin had a 36\% lower risk of all-cause mortality and 39\% lower risk of myocardial infarction respectively compared with those treated conventionally. This risk reduction was especially greater for metformin than insulin or sulphonylurea treatment, despite similar glycemic control [3]. Metformin appears to provide cardiovascular protection beyond blood sugar control. In addition to arterial vascular disease, pathology on venous system is also common in patients with diabetes. Epidemiological 
studies demonstrate an increased risk of deep vein thrombosis and pulmonary embolism among diabetic patients $[4,5]$. Deep venous thrombosis frequently causes limitation on daily activities, while pulmonary embolism may further contribute to a life-threatening condition. The effects of metformin on venous thrombosis in patient with type 2 diabetes have not been previously reported. We hypothesized that metformin may reduce the development of venous thrombosis in patient with type 2 diabetes. Utilizing a nationwide database, we conducted this nonrandomized, pair-matched cohort study to investigate the relationship between metformin therapy and the subsequent development of deep venous thrombosis among patients with type 2 diabetes mellitus.

\section{Methods}

Database

The National Health Insurance program in Taiwan has been operating since 1995 and has enrolled nearly all the inhabitants of Taiwan $(23,074,487$ beneficiaries out of $23,162,123$ inhabitants at the end of 2010). The National Health Insurance Research Database (NHIRD) at the National Health Research Institutes (NHRI) (http://w3. nhri.org.tw/nhird/en/index.htm) in Miao-Li (Taiwan) is in charge of the entire National Health Insurance claims database, and it has published numerous extracted datasets for researchers. The NHRI released a cohort dataset comprising 1,000,000 randomly sampled people who were alive during 1997 and collected all the records of these individuals from 1995 onwards. The database has been confirmed by NHRI to be representative of the Taiwanese population [6]. It is also one of the largest nationwide population-based databases in the world, with more than 1000 scientific articles published using its data [7]. In this cohort dataset, each patient's original identification number has been encrypted to protect privacy. Of note, the encrypting procedure is consistent such that the linkage of claims belonging to the same patient is feasible within the NHIRD datasets. The current study was conducted using HNIRD dataset which contained patients' all medical claim records, including coverage for outpatient, inpatient, emergency, dental, traditional Chinese medicine services, and prescription drugs. Because the NHIRD consists of de-identified secondary data released to the public for research purposes, this study was exempt from full review by the Institutional Review Board. The encrypting procedure is consistent, so the linkage of claims belonging to the same patient is feasible within the NHIRD.

\section{Study sample and control}

We identified patients who used metformin for diabetes treatment (International Classification of Diseases, Ninth Revision, Clinical Modification [ICD-9-CM] codes
250.0 - 250.9) from the 1,000,000 sampling cohort dataset from January 1, 1997. An age-, sex-, and co-morbidity matched non-expose control group was selected from those patients who did not use metformin throughout the whole course of follow-up. To investigate the causal relationship between metformin use and deep venous thrombosis (DVT) occurrence, in both groups, subjects with pre-existing DVT (ICD-9-CM codes 453.0-453.9) before enrollment were excluded from this study. Women with diagnosis of pregnancy during study period were also excluded, since the first-line anti-hyperglycemic agent for gestational diabetes is insulin, not metformin [8]. The co-morbidities to be matched in the 2 groups included pre-existing (upon enrollment) hypertension (ICD-9-CM codes 401.xx-405.xx), coronary artery disease (CAD) (411.xx, 413.xx, 414.xx), hyperlipidemia (272-272.4). Previously well documented risk factors for DVT were recorded, such as cancer (140.xx-199.1), traumatic or pathologic fractures involving spine, pelvis, upper, and lower limbs (733.10-733.19, 800.xx-829.xx), and major cardiothoracic, abdominal, pelvic surgeries and orthopedic surgeries of lower limbs. Concurrent medications for blood sugar control, statin, anti-platelet, anticoagulant agents or hormone replacement therapy were also recorded in our study.

\section{Deep vein thrombosis event measurement}

The end point of the study was defined as occurrence of DVT (ICD-9-CM codes 453.0-453.9). In this database, the ICD codes of DVT and the drug code of metformin did not change throughout the whole follow-up period (1997-2003), assuring the consistency of the disease and medication registry. Similar coding to identify DVT events has also been also used in our previous study [9].

\section{Statistical analysis}

Microsoft SQL Server 2005 (Microsoft Corporation, Redmond, Wash) was used for data management and computing. Statistical analyses were performed utilizing SPSS software (Version 18.0; SPSS, Inc., Chicago, Ill). All data were expressed as mean \pm SD or percentage. Comparisons between the 2 groups were determined by independent Student's $t$ test for continuous variables or Pearson's 2 test, Yates' correction for continuity/Fisher's exact test as appropriate for categorical variables. Survival analysis also was assessed using the Kaplan-Meier method, with the significance based on the log-rank test. Cox proportional hazards models were used to test the association of metformin use with DVT. Statistical significance was inferred at a 2 -sided $P$ value of $<0.05$.

\section{Results}

A total of 7167 type 2 DM patients who used metformin (mean age $57.70 \pm 12.55$ years) were identified from the 
1,000,000 sampling cohort dataset between January 1997 and December 2003. Another 7778 subjects without metformin therapy (mean age $57.72 \pm 13.37$ years) were matched for age, sex, co-morbidities, medications, serving as the control group. The demographic parameters of study subjects are listed in Table 1 . Figure 1 showed the entire flow of study and most enrollees in both groups $(88.1 \%$ in metformin cohort and $88.6 \%$ in the control group) remained active and were followed through the end of the study at the end of the study period (December 31, 2003).

During an average of $3.74 \pm 0.80$ years' follow-up period, there was a significantly lower incidence of DVT development among patients with type 2 diabetes who used metformin, compared with the control group (16 [0.22\%] vs $44[0.56 \%], P<0.001)$. Figure 2 outlines the results of a Kaplan-Meier analysis and the log-rank test, which showed that metformin therapy was associated

\section{Table 1 Baseline characteristics of the study population}

\begin{tabular}{|c|c|c|c|}
\hline & \multicolumn{2}{|c|}{ Metformin } & \multirow[b]{2}{*}{$P$ value } \\
\hline & $\begin{array}{c}\text { No } \\
(\mathrm{N}=7778)\end{array}$ & $\begin{array}{c}\text { Yes } \\
(\mathrm{N}=7167)\end{array}$ & \\
\hline Age, years & $57.72 \pm 13.37$ & $57.70 \pm 12.55$ & 0.937 \\
\hline Male gender & $4095(52.6)$ & $3808(53.1)$ & 0.565 \\
\hline Hypertension & $1885(24.2)$ & $1695(23.7)$ & 0.413 \\
\hline$C A D$ & $671(8.6)$ & $535(7.5)$ & 0.010 \\
\hline Hyperlipidemia & $905(11.6)$ & $849(11.8)$ & 0.708 \\
\hline Atrial fibrillation & $69(0.9)$ & $35(0.5)$ & 0.005 \\
\hline Cancer & $372(3.8)$ & $308(4.3)$ & 0.155 \\
\hline Fracture & $538(6.9)$ & $364(5.1)$ & $<0.001$ \\
\hline Major surgery & $405(5.2)$ & $321(4.5)$ & 0.04 \\
\hline \multicolumn{4}{|l|}{ Medications } \\
\hline Aspirin & $696(8.9)$ & $692(9.7)$ & 0.144 \\
\hline Clopidogrel & $12(0.2)$ & $8(0.1)$ & 0.625 \\
\hline Warfarin & $31(0.4)$ & $36(0.5)$ & 0.409 \\
\hline Statin & $263(3.4)$ & $342(4.8)$ & $<0.001$ \\
\hline HRT & $45(0.6)$ & $54(0.8)$ & 0.203 \\
\hline \multicolumn{4}{|c|}{ Other anti-hyperglycemic agents } \\
\hline SU & $7652(98.4)$ & 6941 (96.8) & $<0.001$ \\
\hline Meglitinide & $2117(27.2)$ & $626(8.7)$ & $<0.001$ \\
\hline a-glucosidase Inhibitor & $2629(33.8)$ & $938(13.1)$ & $<0.001$ \\
\hline TZD & $2663(34.2)$ & 1188 (16.6) & $<0.001$ \\
\hline Insulin & $5377(69.1)$ & $2342(32.7)$ & $<0.001$ \\
\hline Kind of drug used & $2.63 \pm 0.84$ & $2.68 \pm 0.85$ & $<0.001$ \\
\hline
\end{tabular}

Data are the mean \pm SD and $n(\%)$.

CAD indicates coronary artery disease; HRT, hormone replacement therapy; $\mathrm{SU}$, sulfonylurea; TZD, Thiazolidinediones.

Fracture includes traumatic or pathological fracture involving upper or lower limbs, spine and pelvis.

Major surgery includes cardiothoracic, abdominal, pelvic surgery or orthopedic surgery of lower limbs. with a significantly lower incidence of DVT than those without metformin (Log-rank $P=0.001$ ). The significant difference between groups was observed in the first year, even just after six months treatment of metformin. Comparison between patients with and without DVT was shown in Table 2. Patients with DVT were older, more likely to be male, with more co-morbidities of coronary artery disease, fractures and performed more major surgeries.

The Cox proportional hazards regression model was used to determine the factors independently associated with the development of DVT. After adjusting for age, sex, and the aforementioned significant co-morbidities, only age (hazard ratio [HR] 1.031; 95\% CI, 1.009-1.053); $P=0.004$ ), CAD (HR 2.208; 95\%, CI, 1.026-4.751; $P=0.043)$, and major surgery $([\mathrm{HR}] 3.161 ; 95 \% \mathrm{CI}$, $1.535-6.510 ; P=0.002$ ) were independently associated with DVT development, while metformin was independently associated with lower risk of DVT (HR 0.427; 95\%, CI, 0.240-0.758; $P=0.004$ ) (Table 3).

\section{Discussions}

Our major findings of the present study demonstrated metformin use was associated with reduced risk of developing DVT among patients with type $2 \mathrm{DM}$ using a large-scale nationwide database in Asian population. In addition, increasing age and history of cardiovascular disease were found to contribute independently to the risk of DVT.

Metformin is an insulin-sensitizing biguanide used to treat type $2 \mathrm{DM}$. The glucose-lowering effect is a consequence of reduced hepatic gluconeogenesis and increased insulin-stimulated glucose uptake in skeletal muscle and adipocytes $[10,11]$. In addition to the anti-hyperglycemic effects, metformin provides additional cardioprotective effects beyond sugar lowering, which may be related to the actions of metformin on lipid metabolism, vascular smooth muscle and cardiomyocyte intracellular calcium handling, endothelial function, hyper-coagulation and platelet hyperactivity. It also provide greater protection against the development of macrovascular complications than would be expected from its effects on glycemic control alone [12]. There are statistically significant reductions in the risk of all-cause mortality and diabetes-related mortality [3]. Either as monotherapy or in combination with sulfonylurea, metformin was associated with reduced all-cause and cardiovascular mortality compared with sulfonylurea monotherapy [13]. The UKPDS post-trial reported significant and persistent risks reductions for diabetes-related end point, myocardial infarction and death from any cause [14]. Current guidelines from the American Diabetes Association/European Association for the study of Diabetes (ADA/EASD) recommend early initiation of metformin as a first-line drug for 


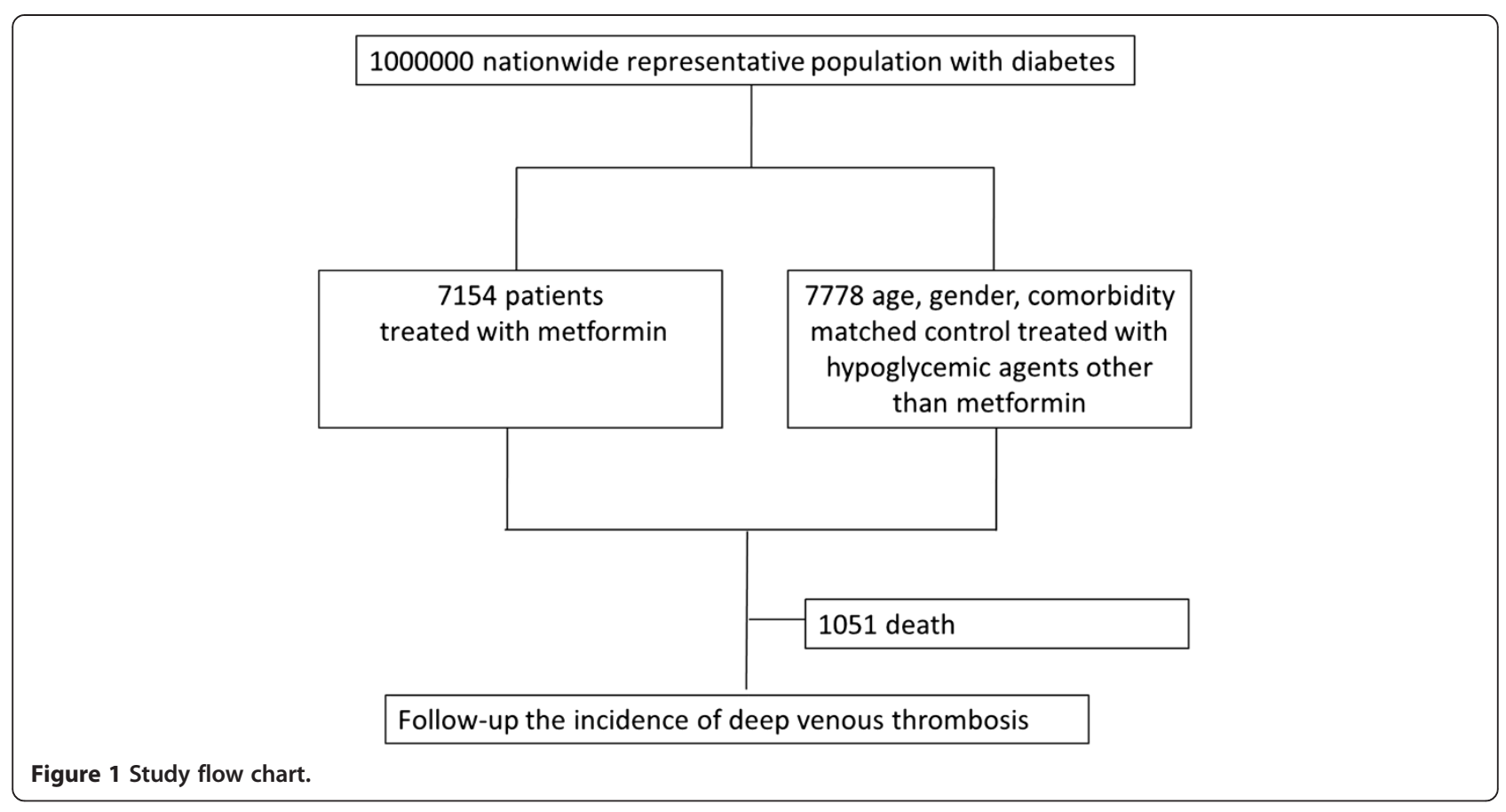

monotherapy and for combination therapy for patients with type 2 diabetes [15].

Our cohort study is the first study to document the protective effect of metformin on venous thromboembolism using a large-scale nationwide cohort database. It is widely accepted that DM impairs endothelial nitric oxide synthase (eNOS) activity and enhances the production of reactive oxygen species (ROS), thus

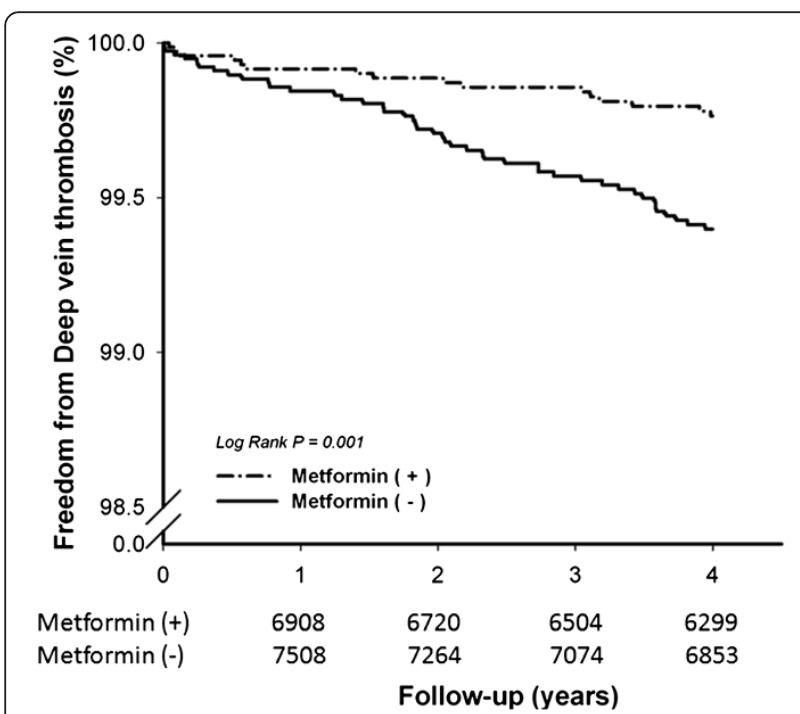

Figure 2 Kaplan-Meier curves revealed patient under metformin therapy was associated with lower incidence of deep vein thrombosis (log-rank test, $p=0.001)$.
Table 2 Baseline characteristics of the DVT population

\begin{tabular}{llll}
\hline & \multicolumn{2}{c}{ DVT } & \\
\cline { 2 - 3 } & No (N=14885) & Yes (N = 60) & P value \\
\hline Age, years & $57.69 \pm 12.98$ & $62.35 \pm 13.31$ & 0.006 \\
Male gender & $7867(52.8)$ & $36(60)$ & 0.328 \\
Hypertension & $3560(23.9)$ & $20(33.3)$ & 0.120 \\
CAD & $1194(8.0)$ & $12(20)$ & 0.003 \\
Hyperlipidemia & $1745(11.7)$ & $9(15)$ & 0.558 \\
Atrial fibrillation & $102(0.7)$ & $2(3.3)$ & 0.065 \\
Cancer & $674(4.5)$ & $680(4.6)$ & 0.054 \\
Fracture & $894(6.0)$ & $8(13.3)$ & 0.027 \\
Major surgery & $716(4.8)$ & $10(16.7)$ & 0.001 \\
\hline Medications & & & \\
Aspirin & $1381(9.3)$ & $7(11.7)$ & 0.679 \\
Clopidogrel & $20(0.1)$ & $0(0.0)$ & 1.000 \\
$\quad$ Warfarin & $63(0.4)$ & $4(6.7)$ & $<0.001$ \\
Statin & $601(4.0)$ & $4(6.7)$ & 0.308 \\
HRT & $99(0.7)$ & $0(0.0)$ & 1.000 \\
Metformin & $7151(48.0)$ & $16(26.7)$ & 0.001 \\
\hline
\end{tabular}

Data are the mean $\pm S D$ and $n(\%)$.

CAD indicates coronary artery disease; HRT, hormone replacement therapy.

Fracture includes traumatic or pathological fracture involving upper or lower limbs, spine and pelvis.

Major surgery includes cardiothoracic, abdominal, pelvic surgery or orthopedic surgery of lower limbs. 
Table 3 Predictors of deep vein thrombosis identified by uni- and multi-variate Cox regression analysis

\begin{tabular}{|c|c|c|c|c|}
\hline & \multicolumn{2}{|l|}{ Univariate analysis } & \multicolumn{2}{|c|}{ Multi-variate analysis } \\
\hline & HR (95\% Cl) & $\mathbf{P}$ & HR $(95 \% \mathrm{Cl})$ & $\mathbf{P}$ \\
\hline ge, per year & $1.032(1.010-1.054)$ & 0.004 & $1.031(1.009-1.053)$ & 0.0 \\
\hline Male gender & $1.361(0.812-2.282)$ & 0.242 & $.934-2.661)$ & 0.088 \\
\hline n & 5) & 2 & 100 & 0.983 \\
\hline CAD & $2.956(1.570-5.564)$ & 0.001 & $2.208(1.026-4.751)$ & 0.043 \\
\hline Hyp & 1.2 & 31 & 4) & 0 \\
\hline Cancer & $2.694(1.159-6.263)$ & 0.021 & $1.598(0.657-3.888)$ & 0.301 \\
\hline act & $2.495(1.185-5.251)$ & 0.016 & $1.918(0.899-4.094)$ & ברפת \\
\hline Major surgery & $4.055(2.057-7.996)$ & $<0.001$ & $3.161(1.535-6.510)$ & 0.002 \\
\hline Aspirin & 1.31 & 0.496 & 0.7 & 0 \\
\hline Dipyridamole & $1.014(0.368-2.797)$ & 0.978 & $0.728(0.257-2.063)$ & 0.550 \\
\hline tatin & $1.667(0.604-4.596)$ & 0.324 & $1.553(0.499-4.836)$ & 0. \\
\hline Metformin & $0.395(0.223-0.700)$ & 0.001 & $0.427(0.240 .0 .758)$ & 0.004 \\
\hline
\end{tabular}

CAD indicates coronary artery disease.

Fracture includes traumatic or pathological fracture involving upper or lower limbs, spine and pelvis.

Major surgery includes cardiothoracic, abdominal, pelvic surgery or orthopedic surgery of lower limbs.

resulting in diminished NO bioavailability and the consequent pro-atherogenetic alterations [16]. Insulin is a normal regulator of eNOS activation and NO production through successive phosphorylation. Insulin resistance in DM attenuates the process and suppresses the normal NO secretion [17]. In previous studies, metformin treatment significantly improved glycation, oxidative stress, nitric oxide (NO) bioavailability and insulin resistance and normalized endothelial function in aorta of rats with diabetes [18]. In human, subjects who received metformin had improvement in endothelium-dependent, acetylcholine-stimulated flows compared with those treated with placebo. In young women with polycystic ovary syndrome, which have an increased prevalence of insulin resistance, short-term metformin therapy improves arterial stiffness and endothelial function [19]. Although the mechanism was still not clear, metformin may help restore endothelial function via modulation of insulin resistance.

It has been reported that metformin treatment was associated with improvements in plasma markers of endothelial function, including von Willebrand factor (vWF), soluble vascular adhesion molecule-1 (sVCAM-1), tissue-type plasminogen activator ( $\mathrm{t}-\mathrm{PA}$ ), plasminogen activator inhibitor-1 (PAI-1) and soluble intercellular adhesion molecule-1 (sICAM-1), suggesting the benefit of endothelial function improvement and inflammation reduction [20]. Metformin have specific effects on endothelial function protection, which explained about $34 \%$ of the reduction in the in the risk of $\mathrm{CV}$ morbidity and mortality [20]. These findings indicate that metformin is able to improve endothelial reactivity at the macro- and microcirculatory level, both of which relate to cardiovascular outcomes [21,22].

Insulin resistance is associated with hypofibrinolysis, and metformin has been shown to improve insulin sensitivity and fibrinolysis [23]. In animal model, metformin prolonged activated partial thromboplastin and prothrombin times, and the endothelial cell damage improved [24]. Sobel et al. demonstrated insulin-sensitizing strategy led to lower fibrinogen level [25]. It may alter fibrin structure and function by interfering with the process involved in fibrin polymerization and lateral aggregation [26]. A reduction in coagulation factor VII levels and factor VIII activity had also been demonstrated $[26,27]$. In subjects with obesity, there was a significantly greater decrease in tissue plasminogen activator (t-PA) antigen and $\mathrm{vWF}$ in the metformin than in the placebo group. The use of metformin increased t-PA activity and decreased t-PA antigen in patients with insulin resistance and hypertension [23,28]. Patients with newly diagnosed type 2 diabetes treated with 8 -week metformin received an intravenous infusion of L-arginine before and after metformin treatment, while L-arginine is the natural precursor of NO and may be useful to assess endothelium-dependent vascular function in humans. The decrease in both platelet aggregation and blood viscosity after L-arginine was significantly amplified after metformin [29]. Therefore insulin sensitizing therapy with metformin may alter the coagulation profiles, which bring beneficial consequences in the thrombogenesis. Together all, metformin may have better endothelial protection and coagulation, which may provide possible mechanisms connected to the reduced DVT events observed in our study.

A number of published studies have shown that the incidence of first-time venous thromboembolism (VTE) rises with age. The incidence increases dramatically after age $60[30,31]$. Although use of oral contraceptives and post-menopausal hormone replacement have been associated with VTE in women, published data suggest no consistent differences in the incidence of VTE among men and women [31]. Besides, we demonstrated that history of cardiovascular disease is associated with development of DVT. In recent years, epidemiological studies have explored the association between VTE, arterial thromboembolism (CVD, MI, stroke) and atherosclerosis, indicating patients with DVT had an increased relative risk for MI and stroke [32]. Since they share several common risk factors, including smoking, immobility, and as our patient group - DM, it is not surprising that a patient with CVD may have increased risk of venous thromboembolism [33].

Previous studies reported metformin treatment was associated with a decrease in vitamin B12 concentration [34], which was present in 5.8\% of the population [35] 
and vitamin B12 deficiency may be associated with hyperhomocysterinemia. However the association between vitamin B12 deficiency and deep vein thrombosis remained undetermined. Previous data have suggested that there is no adequate evidence concerning the role of metformin therapy and hyperhomocysteinemia. A previous study from Thailand demonstrated $\mathrm{s}$ that although metformin may have caused low vitamin B12 levels, there were no significant changes to homocysteine levels [36]. Hoogeveen et al. further found that metformin-exposed patients had only slightly higher serum total homocysteine levels then control group $[37,38]$. Furthermore, the dose-response relationship between cumulative exposure to metformin and total homocysteine level was not identified [38]. Besides medications, other dietary factors such as fruit and vegetable consumption in diabetic patients could also be strong independent determinants of homocysteine levels [39]. Recent studies show that lowering homocysteine levels does not decrease the risk for atherosclerosis or thrombosis $[40,41]$. This supports the theory that homocysteine may just be an "innocent bystander" and not the cause of these conditions. Therefore, large-scale prevention studies identifying high-risk patients through genetic tests, like C677T homozygous mutation targeting on populations with low folate intake have not been performed [42].

In addition, it has been reported that serum vitamin B12 levels do not adequately assess tissue vitamin B12 stores [43]. Patients with type 2 diabetes may show normal extracellular vitamin B12, but disturbed intracellular B12-dependent biochemical reactions. Metformin treatment was associated with low serum vitamin B12 level, while improved intracellular vitamin B12 metabolism despite low serum vitamin B12 [44].

There are some limitations in our study. First, it is a nonrandomized, pair-matched cohort study. The diagnoses of type 2 DM and DVT rely on ICD coding from insurance claim database. The diagnosis of deep venous thrombosis is confirmed by venography or lower limb Doppler ultrasonography, while the arrangement and interpretation of these image studies depend on clinician's judgment. Therefore the incidence of deep venous thrombosis may be underestimated. In addition, some personal information, including smoking, immobilization or concurrent medication was not available in the administrative data. Since smoking, immobility, or use of contraceptive agents are well recognized risk factors of deep vein thrombosis [45]. Accurate assessment of the contributory and confounding effect of these factors are not feasible. Because of the limitation of National Health Insurance Research Database (NHIRD), we were unable to calculate the propensity score as correction of comorbidities. Individual biochemistry data was not available in this population-based registry. Therefore, we cannot measure patients' coagulation and inflammation profiles, and the relationship cannot be delineated. Despite these limitations, this study was believed to give the first insight about the effect of metformin on venous thromboembolism.

\section{Conclusion}

In conclusion, we identified that metformin therapy may be associated with a protective effect in patients with type 2 diabetes mellitus from deep vein thrombosis in a large-scale population-based study. Further larger prospective studies or meta-analysis are needed to confirm our findings.

\section{Abbreviations}

CVD: Cardiovascular disease; DM: Diabetes mellitus; DVT: Deep vein thrombosis; eNOS: Endothelial nitric oxide synthase; ICD: International classification of diseases; NHRI: National Health Research Institutes; NO: Nitric oxide; PAl-1: Plasminogen activator inhibitor-1; PE: Pulmonary embolism; ROS: Reactive oxygen species; sICM-1: Intercellular adhesion molecule-1; sVCAM-1: Soluble vascular adhesion molecule-1; t-PA: Tissue-type plasminogen activator; VTE: Venous thromboembolism; VWF: von Willebrand factor.

\section{Competing interests}

The authors declare that they have no competing interests.

\section{Authors' contributions}

DYL and HBL wrote the study protocol and designed the study. JWC and WLC oversaw the conduct of the study. $\mathrm{CCH}$ and PHH acquired data. CMC performed the statistical analysis. DYL wrote the manuscript. SJL and HBL contributed to the data interpretation reviewed and edited the manuscript. All authors read and approved the final manuscript.

\section{Acknowledgement}

We acknowledge research staff for their valuable assistance.

\section{Author details}

'Division of Cardiology, Department of Medicine, Taipei Veterans General Hospital, Taipei, Taiwan. ${ }^{2}$ Department of Medical Research and Education, Taipei Veterans General Hospital, Taipei, Taiwan. ${ }^{3}$ Cardiovascular Research Center, Taipei Veterans General Hospital, Taipei, Taiwan. ${ }^{4}$ Institute of Pharmacology, Taipei Veterans General Hospital, Taipei, Taiwan. ${ }^{5}$ Institute of Clinical Medicine, National Yang-Ming University, Taipei, Taiwan. ${ }^{6}$ Institute of Biomedical Sciences, Academia Sinica, Taipei, Taiwan. ${ }^{7}$ Healthcare and Management Center, Division of Cardiology, Taipei Veterans General Hospital, 201 Sec. 2, Shih-Pai Road, Taipei, Taiwan.

Received: 29 June 2014 Accepted: 11 December 2014

Published: 15 December 2014

\section{References}

1. Influence of diabetes on 5-year mortality and morbidity in a randomized trial comparing CABG and PTCA in patients with multivessel disease: the Bypass Angioplasty Revascularization Investigation (BARI). Circulation 1997, 96:1761-1769

2. Kirpichnikov D, McFarlane SI, Sowers JR: Metformin: an update. Ann Intern Med 2002, 137:25-33.

3. Effect of intensive blood-glucose control with metformin on complications in overweight patients with type 2 diabetes (UKPDS 34). UK Prospective Diabetes Study (UKPDS) Group. Lancet 1998, 352:854-865.

4. Petrauskiene V, Falk M, Waernbaum I, Norberg M, Eriksson JW: The risk of venous thromboembolism is markedly elevated in patients with diabetes. Diabetologia 2005, 48:1017-1021.

5. Ageno W, Becattini C, Brighton T, Selby R, Kamphuisen PW: Cardiovascular risk factors and venous thromboembolism: a meta-analysis. Circulation 2008, 117:93-102. 
6. National Health Research Institutes: National Health Insurance Research Database (updated 2007). Edited by Institute NHR; 2011. Available at: http://w3.nhri.org.tw/nhird//date_01.html.

7. National Health Insurance Research Database: Research/Publications (updated 2010). Edited by Institutes NHR; 2011. Available at: http://w3.nhri org.tw/nhird/en/Research.html.

8. Crowther CA, Hiller JE, Moss JR, MCPhee AJ, Jeffries WS, Robinson JS, Australian Carbohydrate Intolerance Study in Pregnant Women Trial G: Effect of treatment of gestational diabetes mellitus on pregnancy outcomes. N Engl J Med 2005, 352:2477-2486.

9. Chou KT, Huang CC, Chen YM, Su KC, Shiao GM, Lee YC, Chan WL, Leu HB: Sleep apnea and risk of deep vein thrombosis: a non-randomized, pair-matched cohort study. Am J Med 2012, 125:374-380.

10. Bailey CJ, Turner RC: Metformin. N Engl J Med 1996, 334:574-579.

11. Hundal RS, Krssak M, Dufour S, Laurent D, Lebon V, Chandramouli V, Inzucchi SE, Schumann WC, Petersen KF, Landau BR, Shulman Gl: Mechanism by which metformin reduces glucose production in type 2 diabetes. Diabetes 2000, 49:2063-2069.

12. Rojas LB, Gomes MB: Metformin: an old but still the best treatment for type 2 diabetes. Diabetol Metab Syndr 2013, 5:6.

13. Johnson JA, Majumdar SR, Simpson SH, Toth EL: Decreased mortality associated with the use of metformin compared with sulfonylurea monotherapy in type 2 diabetes. Diabetes Care 2002, 25:2244-2248.

14. Holman RR, Paul SK, Bethel MA, Matthews DR, Neil HA: 10-year follow-up of intensive glucose control in type 2 diabetes. N Engl J Med 2008, 359:1577-1589.

15. American Diabetes A: Standards of medical care in diabetes-2013. Diabetes Care 2013, 36:S11-S66.

16. Tousoulis D, Kampoli AM, Tentolouris C, Papageorgiou N, Stefanadis C: The role of nitric oxide on endothelial function. Curr Vasc Pharmacol 2012, 10:4-18.

17. Capellini VK, Celotto AC, Baldo CF, Olivon VC, Viaro F, Rodrigues AJ, Evora PR: Diabetes and vascular disease: basic concepts of nitric oxide physiology, endothelial dysfunction, oxidative stress and therapeutic possibilities. Curr Vasc Pharmacol 2010, 8:526-544.

18. Sena CM, Matafome P, Louro T, Nunes E, Fernandes R, Seica RM: Metformin restores endothelial function in aorta of diabetic rats. Br J Pharmacol 2011, 163:424-437.

19. Agarwal N, Rice SP, Bolusani H, Luzio SD, Dunseath G, Ludgate M, Rees DA: Metformin reduces arterial stiffness and improves endothelial function in young women with polycystic ovary syndrome: a randomized, placebo-controlled, crossover trial. J Clin Endocrinol Metab 2010, 95:722-730.

20. de Jager J, Kooy A, Schalkwijk C, van der Kolk J, Lehert P, Bets D, Wulffele MG, Donker AJ, Stehouwer CD: Long-term effects of metformin on endothelial function in type 2 diabetes: a randomized controlled trial. J Intern Med 2014, 275:59-70.

21. Lieberman EH, Gerhard MD, Uehata A, Selwyn AP, Ganz P, Yeung AC, Creager MA: Flow-induced vasodilation of the human brachial artery is impaired in patients $<40$ years of age with coronary artery disease. Am J Cardiol 1996, 78:1210-1214

22. Fichtlscherer S, Breuer S, Zeiher AM: Prognostic value of systemic endothelial dysfunction in patients with acute coronary syndromes: further evidence for the existence of the "vulnerable" patient. Circulation 2004, 110:1926-1932.

23. Charles MA, Morange P, Eschwege E, Andre P, Vague P, Juhan-Vague I: Effect of weight change and metformin on fibrinolysis and the von Willebrand factor in obese nondiabetic subjects: the BIGPRO1 Study. Biguanides and the Prevention of the Risk of Obesity. Diabetes Care 1998, 21:1967-1972

24. Ghatak SB, Dhamecha PS, Bhadada SV, Panchal SJ: Investigation of the potential effects of metformin on atherothrombotic risk factors in hyperlipidemic rats. Eur J Pharmacol 2011, 659:213-223.

25. Sobel BE, Hardison RM, Genuth S, Brooks MM, McBane RD 3rd, Schneider DJ, Pratley RE, Huber K, Wolk R, Krishnaswami A, Frye RL, BARI 2D Investigators: Profibrinolytic, antithrombotic, and antiinflammatory effects of an insulinsensitizing strategy in patients in the Bypass Angioplasty Revascularization Investigation 2 Diabetes (BARI 2D) trial. Circulation 2011, 124:695-703.

26. Standeven KF, Ariens RA, Whitaker P, Ashcroft AE, Weisel JW, Grant PJ: The effect of dimethylbiguanide on thrombin activity, FXIII activation, fibrin polymerization, and fibrin clot formation. Diabetes 2002, 51:189-197.

27. Grant PJ: Metformin reduces circulating factor VII concentrations in patients with type 2 diabetes mellitus. Thromb Haemost 1998, 80:209-210.

28. Landin $\mathrm{K}$, Tengborn $\mathrm{L}$, Smith U: Treating insulin resistance in hypertension with metformin reduces both blood pressure and metabolic risk factors. J Intern Med 1991, 229:181-187.
29. Marfella R, Acampora R, Verrazzo G, Ziccardi P, De Rosa N, Giunta R, Giugliano D: Metformin improves hemodynamic and rheological responses to L-arginine in NIDDM patients. Diabetes Care 1996, 19:934-939.

30. Anderson FA Jr, Wheeler HB, Goldberg RJ, Hosmer DW, Patwardhan NA Jovanovic B, Forcier A, Dalen JE: A population-based perspective of the hospital incidence and case-fatality rates of deep vein thrombosis and pulmonary embolism. The Worcester DVT Study. Arch Intern Med 1991, 151:933-938.

31. White $\mathrm{RH}$ : The epidemiology of venous thromboembolism. Circulation 2003, 107:14-18.

32. Lowe GD: Common risk factors for both arterial and venous thrombosis. Br J Haematol 2008, 140:488-495.

33. Sorensen HT, Horvath-Puho E, Pedersen L, Baron JA, Prandoni P: Venous thromboembolism and subsequent hospitalisation due to acute arterial cardiovascular events: a 20-year cohort study. Lancet 2007, 370:1773-1779.

34. de Jager J, Kooy A, Lehert P, Wulffele MG, van der Kolk J, Bets D, Verburg J, Donker AJ, Stehouwer CD: Long term treatment with metformin in patients with type 2 diabetes and risk of vitamin B-12 deficiency: randomised placebo controlled trial. BMJ 2010, 340:c2181

35. Reinstatler L, Qi YP, Williamson RS, Garn JV, Oakley GP Jr: Association of biochemical $B_{12}$ deficiency with metformin therapy and vitamin $B_{12}$ supplements: the National Health and Nutrition Examination Survey, 1999-2006. Diabetes Care 2012, 35:327-333.

36. Pongchaidecha M, Srikusalanukul V, Chattananon A, Tanjariyaporn S: Effect of metformin on plasma homocysteine, vitamin B12 and folic acid: a cross-sectional study in patients with type 2 diabetes mellitus. J Med Assoc Thai 2004, 87:780-787.

37. Sato $Y$, Ouchi K, Funase $Y$, Yamauchi K, Aizawa T: Relationship between metformin use, vitamin B12 deficiency, hyperhomocysteinemia and vascular complications in patients with type 2 diabetes. Endocr J 2013, 60:1275-1280.

38. Hoogeveen EK, Kostense PJ, Jakobs C, Bouter LM, Heine RJ, Stehouwer CD: Does metformin increase the serum total homocysteine level in non-insulin-dependent diabetes mellitus? J Intern Med 1997, 242:389-394.

39. Diakoumopoulou E, Tentolouris N, Kirlaki E, Perrea D, Kitsou E, Psallas M, Doulgerakis D, Katsilambros N: Plasma homocysteine levels in patients with type 2 diabetes in a Mediterranean population: relation with nutritional and other factors. Nutr Metab Caardiovasc Dis 2005, 15:109-117.

40. den Heijer M, Willems HP, Blom HJ, Gerrits WB, Cattaneo M, Eichinger S, Rosendaal FR, Bos GM: Homocysteine lowering by $B$ vitamins and the secondary prevention of deep vein thrombosis and pulmonary embolism: A randomized, placebo-controlled, double-blind trial. Blood 2007, 109:139-144

41. Toole JF, Malinow MR, Chambless LE, Spence JD, Pettigrew LC, Howard VJ, Sides EG, Wang CH, Stampfer M: Lowering homocysteine in patients with ischemic stroke to prevent recurrent stroke, myocardial infarction, and death: the Vitamin Intervention for Stroke Prevention (VISP) randomized controlled trial. JAMA 2004, 291:565-575.

42. Holmes MV, Newcombe P, Hubacek JA, Sofat R, Ricketts SL, Cooper J, Breteler MM, Bautista LE, Sharma P, Whittaker JC, Smeeth L, Fowkes FG, Algra A, Shmeleva V, Szolnoki Z, Roest M, Linnebank M, Zacho J, Nalls MA, Singleton AB, Ferrucci L, Hardy J, Worrall BB, Rich SS, Matarin M, Norman PE, Flicker L, Almeida OP, van Bockxmeer FM, Shimokata $\mathrm{H}$, et al: Effect modification by population dietary folate on the association between MTHFR genotype, homocysteine, and stroke risk: a meta-analysis of genetic studies and randomised trials. Lancet 2011, 378:584-594.

43. Solomon LR: Diabetes as a cause of clinically significant functional cobalamin deficiency. Diabetes Care 2011, 34:1077-1080.

44. Obeid R, Jung J, Falk J, Herrmann W, Geisel J, Friesenhahn-Ochs B, Lammert F, Fassbender K, Kostopoulos P: Serum vitamin B12 not reflecting vitamin B12 status in patients with type 2 diabetes. Biochimie 2013, 95:1056-1061.

45. Pomp ER, Rosendaal FR, Doggen CJ: Smoking increases the risk of venous thrombosis and acts synergistically with oral contraceptive use. Am J Hematol 2008, 83:97-102.

doi:10.1186/1471-2261-14-187

Cite this article as: Lu et al:: Metformin use in patients with type 2 diabetes mellitus is associated with reduced risk of deep vein thrombosis: a non-randomized, pair-matched cohort study. BMC Cardiovascular Disorders 2014 14:187. 